CERN-TH/2002-026

MCTP-02-03

SHEP 02-03

hep-ph/0202100

\title{
Supersymmetric Pati-Salam Models from Intersecting D-Branes
}

\author{
L. L. Everett ${ }^{1,2}$, G. L. Kane ${ }^{2}$, S. F. King ${ }^{3}$, S. Rigolin ${ }^{1,2}$, and Lian-Tao Wang ${ }^{2}$ \\ 1. CERN, TH Division, CH-1211 Geneva 23, Switzerland \\ 2. Michigan Center for Theoretical Physics, Ann Arbor, Michigan, 48109, USA \\ 3. Department of Physics and Astronomy, University of Southampton \\ Southampton, S017 1BJ, UK
}

\begin{abstract}
We explore supersymmetric Type I string-motivated three-family scenarios in which the Standard Model is embedded within two sets of intersecting D branes with $U(1)$-extended Pati-Salam gauge groups. We study a model inspired by the Shiu-Tye Type IIB orientifold, in which a three-family scenario is obtained by assuming that the gauge symmetry breaking takes place in two stages; the Pati-Salam group arises from diagonal breaking of the $U(N)$ gauge groups, which is then broken to the SM gauge group. We investigate the diagonal breaking scenario in detail and find that generically there are difficulties involved in decoupling the exotic Higgs remnants. On the phenomenological side, proper low energy gauge coupling predictions effectively lead to a "single brane" scenario for $M_{\text {String }} \sim M_{U} \simeq 10^{16} \mathrm{GeV}$. The soft parameters in this limit are constrained by a well-known sum rule, leading to a distinctive phenomenological pattern for the low energy mass spectrum.
\end{abstract}




\section{INTRODUCTION}

One of the most important unsolved questions for superstring theory is to determine the way(s) in which it can be connected to our observable, low energy world. The main obstacles have been well-known since the development of perturbative string theory: (i) the tremendous degeneracy of string vacua and (ii) how to break supersymmetry (SUSY) and stabilize moduli while resolving the hierarchy problem. Recent developments in string theory have not suggested definitive resolutions to either issue, although they have provided new settings in which to investigate such questions.

Given the absence of a dynamical principle for selecting the correct string vacuum, one may argue that the question of how the Standard Model (SM) or some plausible extension such as the minimal supersymmetric standard model (MSSM) emerges should be put on hold until string theory dynamics are further understood. We instead suggest that while understanding the dynamics of vacuum selection is indeed the ultimate goal, the answer is not likely to be obtainable solely by studying the formal structure of string theory. It is more likely that progress will follow the historical path, in which low energy data provides invaluable guidance in uncovering the nature of the high energy theory.

In our view it is thus important to consider the phenomenological implications of fourdimensional compactifications of string theory. The Type I string framework has particular interest in that it can provide explicit realizations of the braneworld, both with low fundamental scale of $O(\mathrm{TeV})$ and higher fundamental scales ranging from intermediate $O\left(10^{11}\right.$ $\mathrm{GeV}$ ) to GUT values. Many string constructions have been explored, both with supersymmetry [1] 8] and without [9,10]. The nonsupersymmetric models have quite realistic features, but their stability is in doubt. The supersymmetric constructions have stable D-brane configurations but generically are not phenomenologically appealing. There has recently been progress in model building which has led to more realistic models [8].

In this paper, we study supersymmetric Type I string-motivated models in which the SM gauge group is split between different D-brane sectors. The extension we consider is inspired by an explicit string construction, the Shiu-Tye model [5]. This model has two sets of intersecting D-branes which each have a copy of a $U(1)$-extended Pati-Salam [11] gauge group $U(4)_{c} \times U(2)_{L} \times U(2)_{R}$. The right-handed neutrinos are present as open string states in a natural way (in the same multiplet as the quark and charged lepton singlets) as expected in Pati-Salam models. As in the Shiu-Tye model, the SM gauge group is embedded in a nontrivial way: the Pati-Salam gauge groups which break to the SM are obtained from a diagonal breaking of the gauge groups of the two D-brane sectors. The origin of the three SM families is connected to the gauge embedding; the third family and the electroweak Higgs doublets are open string states from one set of D-branes, while the first and second generations are open string states with endpoints on each of the D-brane sectors.

Such scenarios are interesting because the Pati-Salam framework (which has been extensively studied in the literature) provides a setting in which to explore many phenomenological issues. Nonsupersymmetric Pati-Salam models motivated from D-brane setups have also been recently investigated [12]. We analyze supersymmetric Pati-Salam models motivated from intersecting D-brane scenarios. In doing so, we assume for definiteness that the string scale is approximately equal to the unification scale $M_{\text {String }} \sim M_{U} \simeq 10^{16} \mathrm{GeV}$ (i.e., all compactification radii are small), which is also the Pati-Salam symmetry breaking scale. This 
high string scale is chosen to make contact with the successes of traditional SUSY GUTs; however, it may not be preferred within generic Type I models.円 In this case, obtaining proper low energy values of the gauge couplings effectively leads to a "single brane" model. In this limit the soft terms are constrained by a sum rule well known within perturbative heterotic orbifold models. We explore this scenario within a concrete model (" 4224 ") in which the $U(2)_{L, R}$ arise from one set of branes, while $U(4)_{c}$ is obtained from diagonal breaking of the $U(4)$ groups from each D-brane sector. While the model has interesting features, there are phenomenological difficulties which will be explained in detail.

\section{THEORETICAL FRAMEWORK}

The superstring-motivated models we consider are based on $d=4, N=1$ orientifold compactifications of Type II superstring theory [1-8]. In 16] the general features of this class of models as well as details of the effective action at string tree level of the massless states of the open string sectors are presented.2 We consider scenarios in which the non-Abelian gauge groups of the theory are due to two intersecting (orthogonal) stacks of D five-branes (labeled $5_{1}$ and $5_{2}$ ) located at a single orbifold fixed point.

The chiral matter fields (from which the MSSM matter fields will be obtained) and gauge bosons arise from the open string sectors, while the dilaton, moduli, and graviton fields are in the closed string sector. The open string states are labeled according to the location of the string endpoints on the different D-branes of the theory. These states are classified into two categories; the notation of [16] is used to label them. The first includes open strings which start and end on the same set of branes $C_{j}^{5_{1,2}}$ (where $j$ labels the three complex compact dimensions). These fields are in fundamental or antisymmetric tensor representations of the non-Abelian gauge group of the corresponding set of branes. The second category includes open strings which start and end on different sets of branes $C^{5_{1} 5_{2}}$. These fields are bifundamentals with respect to the two non-Abelian gauge groups. Note that within this class of models, non-Abelian gauge singlet fields with Abelian charges are generically absent (for stacks of D-branes with $U(N)$ gauge groups).

\footnotetext{
${ }^{1}$ The presence of antibranes (hence nonsupersymmetric sectors) may be required to satisfy tadpole/anomaly cancellation constraints [13 15]. The string scale should then be lowered to intermediate values if the SUSY-breaking sector is hidden from the observable brane(s) and lowered to $O(\mathrm{TeV})$ if the SUSY-breaking sector has direct couplings to the observable sector.

${ }^{2}$ Note this effective action was derived using $\mathrm{T}$ duality arguments [16] valid for models with all D-branes are located at a single fixed point (not models for which subsets of branes are located at different fixed points or with nontrivial backgrounds).

${ }^{3}$ Here we shall ignore the presence of other closed string states, the twisted moduli. Such states play interesting roles in the theoretical structure of the model and can lead to distinctive phenomenological signatures (see e.g. [17,15, 18]).
} 
An example of a three family orientifold model with the SM gauge group is the Shiu-Tye model [5], a $Z_{6}$ model with background NS-NS field. In this model, the $5_{1}$ sector has gauge group $U(4)^{(1)} \times U(2)^{(1)} \times U(2)^{(1)}$ and the $5_{2}$ sector has $U(4)^{(2)} \times U(2)^{(2)} \times U(2)^{(2)}$ (the existence of identical gauge groups on each set of branes is guaranteed by $\mathrm{T}$ duality). The gauge group on each brane is a U(1)-extended version of the Pati-Salam model $\left(S U(4)_{c} \times S U(2)_{L} \times\right.$ $\left.S U(2)_{R}\right)$. Recall in the Pati-Salam model, the MSSM states lie in the multiplets $F_{a}=(4,2,1)$ and $\bar{F}_{a}=(\overline{4}, 1,2)$, while the Higgs doublets $H_{u, d}$ lie in the bi-doublet $h=(1,2,2)$. The Higgs fields which break the Pati-Salam gauge group to $S U(3) \times S U(2)_{L} \times U(1)_{Y}$ (in which the hypercharge originates from the breaking of the non-Abelian gauge structure) are a pair of vectorlike states $H=(4,1,2)$ and $\bar{H}=(\overline{4}, 1,2)$. The right-handed neutrinos have a natural origin in the theory as components of the $\bar{F}_{a}$ multiplets.

The three families arise in the Shiu-Tye model in an interesting way. The massless spectrum of this model contains one chiral family associated with each of the two D-brane sectors and two families arising from the intersection of the D-brane sectors. Three family scenarios thus require that the SM gauge group be embedded in a nontrivial way within the two D-brane sectors. The desired embedding is that $U(2)_{L}$ and $U(2)_{R}$ each arise from the diagonal breaking of one set of $U(2)^{(1)}$ and $U(2)^{(2)}$ from the $5_{1}$ and $5_{2}$ sectors. The symmetry breaking is triggered by diagonal vacuum expectation values (VEV's) of bifundamental fields charged with respect to the $U(2)$ groups of each sector. $S U(3)_{c}$ is obtained from (say) the breaking of $U(4)^{(1)}$, such that the third family arises from the $5_{1}$ sector.

In the Shiu-Tye model, the doubling of the intersection states is connected with the nonvanishing quantized background NS-NS B field. The presence of the background B field modifies the vertex operators of the intersection states, allowing for a nontrivial multiplicity of states. Such states are not identical from the worldsheet point of view and the selection rules which govern their couplings should be different from those presented for orientifolds without nontrivial background field VEV's in [2.16]. Given the class of available results we shall use the effective action which treats these two families identically, but caution the reader that this symmetry is not likely to hold to all orders in the effective action.

\section{4224 MODEL}

Motivated by the Shiu-Tye model, we consider a different embedding of the SM gauge group from intersecting five-branes with Pati-Salam gauge groups. The gauge group of the $5_{1}$ sector is $U(4)^{(1)} \times U(2)_{L} \times U(2)_{R}$ and the gauge group of the $5_{2}$ sector is $U(4)^{(2)}$ (e.g., we assume the $U(2)$ gauge groups of the $5_{2}$ sector are broken). In this " 4224 " model, the third family arises from the $5_{1}$ sector, while the first and second families are intersection states. The particle content is given in Table I.

Let us first discuss the additional $U(1)$ factors. A close inspection of Table I shows that all but one linear combination of the $U(1)^{\prime}$ 's $\left(U(1)^{\prime}=Q_{4}^{1}+Q_{4}^{2}+Q_{2 L}+Q_{2 R}\right)$ are anomalous (see also [12]) and will be broken at the string scale by the 4D Green Schwarz mechanism of Type I 19. In the 4224 model all of the SM fields listed in Table I as well as the Higgs

\footnotetext{
${ }^{4}$ In contrast to the perturbative heterotic models which have at most one anomalous $U(1)$ since
} 


\begin{tabular}{|c|c|c|c|c|c|c|c|c|c|}
\hline & $S U(4)^{(1)}$ & $S U(2)_{L}$ & $S U(2)_{R}$ & $S U(4)^{(2)}$ & $Q_{4}^{(1)}$ & $Q_{2 L}$ & $Q_{2 R}$ & $Q_{4}^{(2)}$ & \\
\hline$h$ & 1 & 2 & 2 & 1 & 0 & 1 & -1 & 0 & $C_{1}^{5_{1}}$ \\
\hline$F_{3}$ & 4 & 2 & 1 & 1 & 1 & -1 & 0 & 0 & $C_{2}^{5_{1}}$ \\
\hline $\bar{F}_{3}$ & $\overline{4}$ & 1 & 2 & 1 & -1 & 0 & 1 & 0 & $C_{3}^{5_{1}}$ \\
\hline$F_{1,2}$ & 1 & 2 & 1 & 4 & 0 & -1 & 0 & 1 & $C^{55_{1}}$ \\
\hline $\bar{F}_{1,2}$ & 1 & 1 & 2 & $\overline{4}$ & 0 & 0 & 1 & -1 & $C^{5_{1} 5_{2}}$ \\
\hline$H$ & 4 & 1 & 2 & 1 & 1 & 0 & -1 & 0 & $C_{1}^{5_{1}}$ \\
\hline $\bar{H}$ & $\overline{4}$ & 1 & 2 & 1 & -1 & 0 & 1 & 0 & $C_{2}^{5_{1}}$ \\
\hline$\varphi_{1}$ & 4 & 1 & 1 & $\overline{4}$ & 1 & 0 & 0 & -1 & $C^{55_{1} 5_{2}}$ \\
\hline$\varphi_{2}$ & $\overline{4}$ & 1 & 1 & 4 & -1 & 0 & 0 & 1 & $C^{5_{1} 5_{2}}$ \\
\hline$D_{6}^{(+)}$ & 6 & 1 & 1 & 1 & 2 & 0 & 0 & 0 & $C_{1}^{5_{1}}$ \\
\hline$D_{6}^{(-)}$ & 6 & 1 & 1 & 1 & -2 & 0 & 0 & 0 & $C_{2}^{5_{1}}$ \\
\hline
\end{tabular}

TABLE I. The particle content of the 4224 model.

fields $\left\{H, \bar{H}, \varphi_{1,2}\right\}$ are neutral under the persisting nonanomalous $U(1)^{\prime}$. The only fields charged under this $U(1)$ are the $D_{6}$ fields needed to decouple the Higgs triplets after the Pati-Salam gauge symmetry breaking. As these fields must acquire superheavy masses to avoid fast proton decay, this $U(1)^{\prime}$ (although it remains unbroken unless additional fields are introduced into the model to break it) does not have an important impact on the low energy phenomenology. However, the presence of the $U(1)$ factors at the string scale requires that the effective action respect $U(N)$ symmetry rather than $S U(N)$; i.e. the $U(1)$ 's persist as global symmetries. These effective global symmetries have important consequences. For example [5,12], within the $U(1)$-extended Pati-Salam model one of the surviving global symmetries is $U(1)_{B}$, ensuring the stability of the proton even with low fundamental scales (assuming effects of the colored Higgses are suppressed, which will be shown to be a difficult task in the next subsection).

\section{A. Gauge Symmetry Breaking and Decoupling of Exotic Matter}

The gauge symmetry breaking pattern of this model follows the three-family approach of Shiu and Tye [5]. The symmetry breaking takes place in two stages, which are assumed to occur at very similar scales $\sim M_{U}$. In the first stage, $U(4)^{(1)} \times U(4)^{(2)}$ are broken to the diagonal $U(4)$ subgroup (which is identified as $U(4)_{c}$ ) by diagonal VEV's of the bifundamental fields $\varphi_{1,2}$. The resulting theory is an effective Pati-Salam model (with additional $U(1)$ 's) which then breaks to the MSSM (and additional $U(1)$ 's, which we shall ignore) through the

it is the dilaton which participates in the anomaly cancellation, within Type I models there can be multiple anomalous $U(1)$ 's which are cancelled by shifts in the twisted moduli fields. 
Higgs pair of bifundamentals $H, \bar{H}$. To preserve supersymmetry, the symmetry breaking should occur along flat directions; D flatness can be spoiled only by terms of the order of the soft supersymmetry breaking masses. Specifically, the symmetry breaking occurs as follows:

$$
\begin{aligned}
U(4)^{(1)} \times U(4)^{(2)} \times U(2)_{L} \times U(2)_{R} \stackrel{\left\langle\varphi_{1,2}\right\rangle}{\longrightarrow} U(4)_{c} \times U(2)_{L} \times U(2)_{R} \\
\stackrel{\langle H, \bar{H}\rangle}{\longrightarrow} S U(3)_{C} \times S U(2)_{L} \times U(1)_{Y} \times U(1)^{3} .
\end{aligned}
$$

It is instructive to consider this symmetry breaking pattern in further detail and investigate the decoupling of the exotic matter present in the Higgs representations.

Let us first consider the diagonal breaking step, $U(4) \times U(4) \rightarrow U(4)_{c}$. This is achieved when the bi-fundamental fields $\varphi_{1}$ and $\varphi_{2}$ acquire diagonal VEV's

$$
\left\langle\left(\varphi_{1}\right)_{\alpha a}\right\rangle=\delta_{\alpha a} v_{1} ;\left\langle\left(\varphi_{2}\right)_{\alpha a}\right\rangle=\delta_{\alpha a} v_{2},
$$

in which $\alpha$ denotes the $U(4)^{(1)}$ gauge index and $a$ that of $U(4)^{(2)}$. D flatness (particularly for the $U(1)$ generators of the $U(4)$ gauge groups) requires that $v_{1}=v_{2}\left(\sim M_{U}\right)$.

Setting the effects of the soft supersymmetry breaking to zero for the moment, one can easily understand the resulting pattern of Higgs masses. As the gauge symmetry is broken down to the diagonal subgroup but supersymmetry is preserved, the supersymmetric completion of the Higgs mechanism requires that the resulting fields arrange themselves into supermultiplets. There are 16 broken generators of the gauge group, while the states $\varphi_{1,2}$ have a total of 32 (complex) degrees of freedom. Of these 32 complex degrees of freedom in the Higgs sector, 16 real degrees of freedom are Goldstone bosons eaten to form the longitudinal components of the massive gauge bosons. 16 additional real degrees of freedom in the Higgs sector also obtain masses via $\mathrm{D}$ terms. These fields are degenerate in mass with the gauge bosons and together with the 16 massive fermions (with masses due to gauginoHiggsino mixing) form a massive vector supermultiplet. Therefore, 16 complex degrees of freedom remain massless; one real degree of freedom corresponds to the flat direction and there is also another Goldstone boson corresponding to the global $U(1)$ symmetry which remains from the freedom to perform global phase rotations on the fields $\varphi_{1,2}$ (the Goldstone boson of the other global $U(1)$ is eaten to form one of the massive gauge bosons). The other massless fields organize themselves into an adjoint representation of $S U(4)_{c}$, which consists of a color octet, two color triplets, and one color singlet with respect to $S U(3)_{c}$. If such fields remain massless, the proton is not stable. This would certainly rule out this model and suggests serious difficulties with this intersecting brane approach.

In principle, a potential could be constructed utilizing higher-dimensional operators which could presumably decouple these additional states (as well as stabilize the VEV's at the scale $M_{U}$ ). This is beyond the scope of this paper. It is worth noting that if the

\footnotetext{
${ }^{5}$ The $\mathrm{D}$ flatness conditions can be modified if the associated $U(1)$ 's are anomalous. In this case one can choose to resolve or "blow up" the orbifold singularities by giving VEV's to the twisted moduli [19], which induces nonzero Fayet-Iliopoulos D terms. This highly model-dependent option is not considered further in this paper.
} 
gauge groups were $S U(N)$ rather than $U(N)$, D flatness only requires one Higgs field rather than the vectorlike pair (of course anomaly requirements would require additional states in that case). The number of degrees of freedom in the Higgs sector is then halved and there is a desirable matching of the number of Higgs fields to the number of broken generators. p

In the second stage of symmetry breaking, the additional $U(1)$ 's do not complicate the D flatness analysis. However, complications arise for decoupling the additional color triplets from $H, \bar{H}$ after the Pati-Salam symmetry breaking. The standard mechanism [21] of utilizing a single $S U(4)$ sixplet field $D_{6}$ which couples to $H, \bar{H}$ via $W=D_{6} H H+D_{6} \bar{H} \bar{H}$ naively does not work because the necessary operators (for one of the terms) are forbidden by $U(4)$ gauge invariance (due to the $U(1)$ charges, as the $D_{6}$ field can either have $U(1)$ charge \pm 2 ). This problem could be solved via the introduction of non-Abelian singlet fields which are charged under the $U(1)$ 's and acquire string-scale VEV's. However, such fields are not expected in orientifold models when the D-branes are all located at a single fixed point. One can introduce both $D_{6}^{(+)}$and $D_{6}^{(-)}$, in which case gauge invariance allows $D_{6}^{(-)} H H$ and $D_{6}^{(+)} \bar{H} \bar{H}$ (although string selection rules still forbid these terms at trilinear order). However, this will not eliminate massless color triplets, as another color triplet pair has been introduced that remains massless barring the presence of additional couplings.

In the 4224 setup, the D-brane assignments of $H$ and $\bar{H}$ also forbid a trilinear coupling with a $U(4)$ sixplet field $D_{6}$ from the $5_{1}$ brane sector (as the only string-allowed coupling is of the form $\left.C_{1}^{5_{1}} C_{2}^{5_{1}} C_{3}^{5_{1}}\right)$. This problem could be solved if the fields which break the PatiSalam gauge groups are chosen to be a pair of $C^{5_{1} 5_{2}}$ states $H^{\prime}=(1,1, \overline{2}, 4), \bar{H}^{\prime}=(1,1,2, \overline{4})$. In this case the $D_{6}^{\prime}=(1,1,1,6)$ field can be chosen as a $C_{3}^{5_{2}}$ field, which leads to a stringallowed trilinear coupling $C_{3}^{5_{2}} C^{5_{1} 5_{2}} C^{5_{1} 5_{2}}$. However, in this case the baryon number violating couplings $F F D_{6}$ and $\bar{F} \bar{F} D_{6}$ would be equally allowed. In principle, both sets of Higgs pairs $H, \bar{H}, H^{\prime}, \bar{H}^{\prime}$ could be present. In this case, the number of Higgs fields needed to break the same symmetry group has been doubled. This again leads to further massless states (additional color triplets of both the up and down type).

To summarize, it is clear that this model suffers from a major drawback in that it is difficult to decouple the exotic states. As many of these states are charged under $S U(3)_{c}$, they must acquire superheavy masses or they can lead to rapid proton decay. In this setup the additional $U(1)$ 's are the source of the trouble. This may have implications for such intersecting D-brane setups, since the $U(N)$ (rather than $S U(N)$ ) groups are generic to D-branes. The additional $U(1)$ 's make their presence felt in two ways. First, D flatness conditions necessarily lead to a doubling of the Higgs sector in the diagonal breaking step. Second, $U(1)$ gauge invariance (and worldsheet selection rules) can forbid the necessary

\footnotetext{
${ }^{6}$ This can also be understood using the well-known correspondence between holomorphic gauge invariant polynomials and D flat directions [20]. In the $S U(N)$ case the invariant polynomial is given by $\varphi^{N}$, where $\varphi$ is the bifundamental field with the diagonal VEV. In the $U(N)$ case gauge invariance forbids this term and requires the existence of an oppositely charged scalar $\bar{\varphi}$, such that the holomorphic gauge invariant polynomial is $\varphi \bar{\varphi}$.

${ }^{7}$ A global $R$ symmetry is typically utilized to forbid such terms [22].
} 
couplings required to generate masses for the additional states. The issues outlined above for the 4224 model will hold for generic supersymmetric setups with intersecting branes on orbifolds. Similar issues occur within the Shiu-Tye model, although there the exotic states from the diagonal breaking are charged under $S U(2)_{L}$ and $U(1)_{Y}$ rather than $S U(3)_{c}$.

Having noted this generic (and problematic) feature, in the rest of the paper we proceed to study the effective action and phenomenology of the MSSM sector of the 4224 model. In other words, we assume that mechanisms exist which decouple the unwanted exotic states at the string scale. We then study the "observable" sector assuming that only the MSSM states are present below the string scale.

\section{B. Effective Action of MSSM Fields}

We now discuss the form of the effective action of the MSSM sector of the 4224 model, beginning with the gauge couplings. The symmetry breaking pattern leads to the following relations among the gauge couplings of the SM gauge groups in terms of the gauge couplings $g_{5_{1}}$ and $g_{5_{2}}$ associated with the $5_{1}$ and $5_{2}$ sectors (see also [0]):

$$
\begin{aligned}
g_{3} & =\frac{g_{5_{1}} g_{5_{2}}}{\sqrt{g_{5_{1}}^{2}+g_{5_{2}}^{2}}}=g_{4} \\
g_{2} & =g_{5_{1}}=g_{2 R} \\
g_{Y} & =\frac{\sqrt{3} g_{3} g_{2}}{\sqrt{3 g_{3}^{2}+2 g_{2}^{2}}} .
\end{aligned}
$$

The gauge couplings are not unified when $g_{5_{1}}$ and $g_{5_{2}}$ are unrelated, as discussed below.

We now turn to the structure of the superpotential Yukawa couplings. The gauge and Dbrane assignments of the model are designed to allow the $O(1)$ Yukawa coupling of the third family $F_{3} \bar{F}_{3} h$, as $C_{1}^{5_{1}} C_{2}^{5_{1}} C_{3}^{5_{1}}$ is allowed by worldsheet symmetries. The model as expected predicts third family Yukawa unification, such that large of $\tan \beta$ is required to ensure the correct $m_{t} / m_{b}$ mass ratio. The first and second generations do not have large Yukawa couplings at trilinear order as required by phenomenology. The selection rules presented in 116 dictate that $F_{1,2} \bar{F}_{1,2} h$ is forbidden with our choice of the Higgs D-brane assignment. Gauge invariance also forbids terms of the form $F_{1,2} \bar{F}_{3} h$ and $F_{3} \bar{F}_{1,2} h$.

Therefore, the small Yukawa couplings of the first and second families as well as the Majorana mass terms for the neutrinos must therefore be generated via higher-dimensional operators. In [23] these entries in the Yukawa matrix were generated by operators of the

form $F_{i} \bar{F}_{j} h(H \bar{H})^{n}$. In the present model there can be two pairs of heavy Higgs fields $\left\{H, \bar{H}, H^{\prime}, \bar{H}^{\prime}\right\}$. The operators $F_{1,2} \bar{F}_{3} h\left(H \bar{H}^{\prime}\right)$ are allowed by gauge invariance. If they are permitted by worldsheet symmetries, the Clebsch structure could allow for a large 23 entry in the neutrino Yukawa matrix and simultaneously small 23 entries in the other Yukawa matrices, allowing for a large 23 neutrino mixing angle [25]. The small 13 mixing angles would suggest that the 13 operators are all suppressed by some additional symmetry which plays the role of the $U(1)$ family symmetry in [25]. It is also unclear whether unsuppressed operators such as $F_{1,2} \bar{F}_{1,2} h(H \bar{H})$ are permitted. Note that the addition of the two pairs of Higgs fields needed for interesting lepton textures lead to additional color triplet states 
which remain massless after the symmetry breaking unless additional higher-dimensional operators are introduced to decouple them.

Thus, such intersecting D-brane models potentially have a rich flavor structure, although the details of the higher-dimensional operators required remain speculative. To determine whether any flavor structure is viable will of course depend in detail on these smaller Yukawa couplings (and the associated soft trilinear couplings). Despite these limitations, it is worth elaborating on certain aspects of the flavor structure which might be expected. As stated, the 33 Yukawa entry gets a large tree level contribution while the other entries are only generated by higher order operators. Assuming that the first two generations are governed by the same selection rules at leading order, the Yukawas would take the following approximate form:

$$
Y_{i j} \simeq\left[\begin{array}{lll}
a & a & b \\
a & a & b \\
c & c & 1
\end{array}\right]
$$

where $a, b$ and $c$ are generated by higher-dimensional operators. Given the assumed (near) degeneracy of the first and second generation gauge eigenstates, a hierarchy of fermion masses can naturally be generated within this framework.

At this stage we can write down the soft parameters of the 4224 model, using the phenomenological approach of [26]. [1 We use the notation

$$
\begin{aligned}
F^{S} & =\sqrt{3}\left(S+S^{*}\right) m_{3 / 2} X_{0} e^{i \alpha_{S}} \\
F^{i} & =\sqrt{3}\left(T_{i}+T_{i}^{*}\right) m_{3 / 2} X_{i} e^{i \alpha_{i}},
\end{aligned}
$$

in which $m_{3 / 2}$ is the gravitino mass and $X_{0, i=1,2,3}$ measure the relative contributions of $S$ and $T_{i}$ to the supersymmetry breaking (the $X_{i}$ are related to the usual Goldstino angle parameterization [26, 16] via $\left.X_{0} \equiv \sin \theta, X_{i} \equiv \cos \theta \Theta_{i}\right)$. The $X_{i}$ satisfy the relation $\sum_{i} X_{i}^{2}=$ 1. In general, the $F$ - component VEV's can have arbitrary complex phases $\alpha_{S}, \alpha_{i}$. The gaugino masses of the Pati-Salam gauge groups are given by:

$$
\begin{aligned}
& m_{4}^{(1)}=\sqrt{3} m_{3 / 2} X_{1} e^{-i \alpha_{1}}=m_{2 L}=m_{2 R} \\
& m_{4}^{(2)}=\sqrt{3} m_{3 / 2} X_{2} e^{-i \alpha_{2}},
\end{aligned}
$$

such that the MSSM gaugino masses are

\footnotetext{
${ }^{8}$ Within this approach (with a high string scale $M_{\text {String }} \sim M_{U} \sim 10^{16} \mathrm{GeV}$ ), the presence of a hidden (which communicates with the observable sector only gravitationally) or sequestered sector is assumed in which the $N=1$ SUSY present at the string scale is broken by some unknown dynamical mechanism (i.e. gaugino condensation) within the effective field theory. We do not attempt to model this sector in this paper. However, if all the branes within the theory are assumed to sit on a single fixed point, there is no truly sequestered sector. It is not known whether potentially realistic orientifold models with $N=1$ SUSY can be constructed which have these relevant features (as discussed in [14]).
} 


$$
\begin{aligned}
m_{3} & =\frac{\sqrt{3} m_{3 / 2}}{\left(T_{1}+T_{1}^{*}\right)+\left(T_{2}+T_{2}^{*}\right)}\left[\left(T_{1}+T_{1}^{*}\right) X_{1} e^{-i \alpha_{1}}+\left(T_{2}+T_{2}^{*}\right) X_{2} e^{-i \alpha_{2}}\right] \\
m_{2} & =\sqrt{3} m_{3 / 2} X_{1} e^{-i \alpha_{1}} \\
m_{1} & =\frac{\sqrt{3} m_{3 / 2}}{\frac{5}{3}\left(T_{1}+T_{1}^{*}\right)+\frac{2}{3}\left(T_{2}+T_{2}^{*}\right)}\left[\frac{5}{3}\left(T_{1}+T_{1}^{*}\right) X_{1} e^{-i \alpha_{1}}+\frac{2}{3}\left(T_{2}+T_{2}^{*}\right) X_{2} e^{-i \alpha_{2}}\right] .
\end{aligned}
$$

The soft mass-squared parameters are given by

$$
\begin{aligned}
m_{h}^{2} & =m_{H_{u}}^{2}=m_{H_{d}}^{2}=m_{3 / 2}^{2}\left(1-3 X_{0}^{2}\right) \\
m_{F_{3}}^{2} & =m_{Q_{3}}^{2}=m_{L_{3}}^{2}=m_{3 / 2}^{2}\left(1-3 X_{3}^{2}\right) \\
m_{\bar{F}_{3}}^{2} & =m_{U_{3}}^{2}=m_{D_{3}}^{2}=m_{E_{3}}^{2}=m_{3 / 2}^{2}\left(1-3 X_{2}^{2}\right) \\
m_{F_{1,2}}^{2} & =m_{Q_{1,2}}^{2}=m_{L_{1,2}}^{2}=m_{3 / 2}^{2}\left(1-\frac{3}{2}\left(X_{0}^{2}+X_{3}^{2}\right)\right) \\
m_{\bar{F}_{1,2}}^{2} & =m_{U_{1,2}}^{2}=m_{D_{1,2}}^{2}=m_{E_{1,2}}^{2}=m_{3 / 2}^{2}\left(1-\frac{3}{2}\left(X_{0}^{2}+X_{3}^{2}\right)\right) .
\end{aligned}
$$

The Yukawa coupling of the third family is present in the superpotential at trilinear order and hence there is a prediction for the associated 33 entry of the trilinear coupling $\tilde{A}$ :

$$
\tilde{A}_{33}=-\sqrt{3} m_{3 / 2} X_{1} e^{-i \alpha_{1}} Y_{33}
$$

In the absence of a complete theory for the smaller Yukawa couplings it is not possible to predict the structure of the associated soft trilinear couplings. However, we can speculate on their form assuming that the smaller Yukawas have been generated at the unification scale and can be treated as effective trilinear superpotential couplings (as in e.g. [27]). f] In this case, the soft trilinear couplings take the form

$$
\tilde{A}_{i j}=\left[\begin{array}{lll}
d_{4} Y_{11} & d_{4} Y_{12} & d_{2} Y_{13} \\
d_{4} Y_{21} & d_{4} Y_{22} & d_{2} Y_{23} \\
d_{3} Y_{31} & d_{3} Y_{32} & d_{1} Y_{33}
\end{array}\right],
$$

with

$$
\begin{aligned}
& d_{1}=-\sqrt{3} m_{3 / 2} X_{1} e^{-i \alpha_{1}}=-M_{2} \\
& d_{2}=\sqrt{3} m_{3 / 2}\left(\frac{1}{2} X_{0} e^{-i \alpha_{0}}-\frac{1}{2} X_{3} e^{-i \alpha_{3}}\right)+d_{1} \\
& d_{3}=\sqrt{3} m_{3 / 2}\left(\frac{1}{2} X_{0} e^{-i \alpha_{0}}-X_{2} e^{-i \alpha_{2}}+\frac{1}{2} X_{3} e^{-i \alpha_{3}}\right)+d_{1} \\
& d_{4}=\sqrt{3} m_{3 / 2}\left(X_{0} e^{-i \alpha_{0}}-X_{2} e^{-i \alpha_{2}}\right)+d_{1} .
\end{aligned}
$$

From Eq. (14), the trilinear soft terms have a form similar to Eq. (4). The pattern of soft trilinear couplings described above is similar to perturbative heterotic orbifold stringmotivated models with matter in the twisted sector [29.

\footnotetext{
${ }^{9}$ See [28] for a more complete approach in which the small Yukawas as well as the soft trilinears are determined within perturbative heterotic string models.
} 
The supersymmetric Higgs mass parameter $\mu$ is absent in the string-derived superpotential. In principle, $\mu$ and and the associated soft parameter $b$ must be generated in the effective theory (e.g. by the Giudice-Masiero mechanism [30]). As this is model-dependent, we prefer to leave them as free parameters in the phenomenological analysis (their magnitudes will be fixed by the requirement of radiative electroweak symmetry breaking).

\section{PHENOMENOLOGICAL IMPLICATIONS}

\section{A. Approximate Unification of Gauge Couplings}

The gauge couplings of string models are related to the size and shape of the compactification manifold. Thus, the experimental measurements of the gauge couplings provide information about the nature of the compactification of a specific model. It is important to check that the phenomenological requirements on the gauge couplings do not introduce any inconsistencies. Specifically, the mass scales associated with the Kaluza-Klein (KK) and winding states introduce corrections to the running of the gauge couplings. As the 4224 symmetry group breaks at the traditional unification scale $M_{U} \sim 10^{16} \mathrm{GeV}$, we choose to identify the lowest scale as $M_{U}$ so that the usual RG running in the $d=4$ effective field theory can be applied. The gauge couplings of the five-branes are given by

$$
g_{5_{i}}^{2}=\frac{4 \pi}{R e T_{i}}=\frac{4 \pi \lambda_{I} M_{i}^{2}}{2 M_{I}^{2}}
$$

where $M_{i} \propto 1 / R_{i}$ characterizes the compactification size of the $i$ th compact complex dimension, $\lambda_{I}$ is the Type I string coupling, and $M_{I}$ is the Type I string scale. Defining $g_{5_{2}}=a g_{5_{1}}$, we obtain a relation between the compactification size of the two intersecting branes:

$$
\frac{M_{1}}{M_{2}}=\frac{R_{2}}{R_{1}}=\frac{1}{a} .
$$

In the 4224 model, the gauge couplings of the Standard Model gauge group near the unification scale are given by Eq. (3):

$$
\begin{aligned}
g_{3}^{2} & =\frac{g_{5_{1}}^{2} g_{5_{2}}^{2}}{g_{5_{1}}^{2}+g_{5_{2}}^{2}}=\frac{g_{2}^{2}}{1+1 / a^{2}} \\
g_{2}^{2} & =g_{5_{1}}^{2} \\
g_{Y}^{2} & =\frac{3 g_{5_{1}}^{2} g_{5_{2}}^{2}}{3 g_{5_{1}}^{2}+2 g_{5_{2}}^{2}}=\frac{3}{5} \frac{g_{2}^{2}}{1+2 / 5 a^{2}} .
\end{aligned}
$$

The gauge couplings are only unified in the $a \rightarrow \infty$ limit such that $T_{2} \ll T_{1}\left(R_{2} \ll R_{1}\right)$. This corresponds to a "single brane" limit in which the $5_{2}$ gauge sector decouples from the low energy physics, as can be seen from Eq. (7). We can investigate approximate gauge coupling unification by choosing $a^{2}$ rather large. This effective "single brane" scenario will have important phenomenological implications on the low energy spectrum of the model.

Notice that Eq. (19) is valid only at the string scale, while the unification condition should be imposed at the unification scale. For self-consistency, it is necessary that the 
string and unification scales are very close to each other, such that the RG running does not significantly modify the ratio of the gauge couplings. An additional constraint on the model is the usual requirement that the theory remains in the perturbative region $\left(\lambda_{I}<1\right)$. As an example, we present a simple self-consistent realization. Consider the case in which all inverse radii except for $R_{2} \equiv 1 / M_{2}$ are equal:

$$
M_{1}=\frac{M_{2}}{a}=M_{3}=M_{c}
$$

Using the relations between the Planck scale, string scale, and compactification scales in Type I theory [16], one can obtain [31] the useful relation $\alpha_{5_{i}} M_{P} / \sqrt{2}=M_{I}^{2} M_{i} /\left(M_{j} M_{k}\right)$ $(i \neq j \neq k)$. Using this result and Eq. (22), the string scale and compactification scale $M_{c}$ are related by

$$
\frac{a M_{I}^{2}}{M_{c}} \simeq 3.5 \times 10^{17} \mathrm{GeV} .
$$

Now consider the case in which $M_{c}<M_{I} / \sqrt{a}$. The lowest scale is a winding mode with mass $M_{c}$ and thus we identify $M_{c}=M_{U}=3 \times 10^{16} \mathrm{GeV}$. From Eq. (23), the string scale (for $a=4$ ) is $M_{I}=5 \times 10^{16} \mathrm{GeV}$. The theory remains in the perturbative regime, as $\lambda_{I}=2 \sqrt{2} M_{I}^{4} /\left(a M_{c}^{3} M_{P}\right)<1$. Therefore, this approximate unification approach is consistent and could in principle be realized in simple orbifold models. T

Let us now investigate the implications of the approximate unification scenario (see [12] for a more detailed analysis for the nonsupersymmetric Pati-Salam brane models). First, Eq. (21) reduces $g_{3}$ compared to the naive unification, which is the direction required by experiment.⿴囗十丁 More precisely,

$$
\begin{aligned}
\sin ^{2}\left(\theta_{W}\right)\left(M_{Z}\right) & =\frac{3}{8} \frac{1}{1+1 / 4 a^{2}}\left(1-\frac{\alpha\left(M_{Z}\right)}{2 \pi}\left(b_{Y}+b_{2}\right) \log \frac{M_{U}}{M_{Z}}\right)+\frac{\alpha\left(M_{Z}\right)}{2 \pi} b_{2} \log \frac{M_{U}}{M_{Z}} \\
\frac{1}{\alpha_{s}\left(M_{Z}\right)} & =\frac{3}{8} \frac{1+1 / a^{2}}{1+1 / 4 a^{2}}\left(\frac{1}{\alpha\left(M_{Z}\right)}-\frac{1}{2 \pi}\left(b_{Y}+b_{2}\right) \log \frac{M_{U}}{M_{Z}}\right)+\frac{1}{2 \pi} b_{3} \log \frac{M_{U}}{M_{Z}},
\end{aligned}
$$

in which the MSSM beta functions are $b_{Y}=11, b_{2}=1, b_{3}=-3$. The standard unification picture is reproduced for larger values of $a$, but $a$ is bounded by the requirement that the gauge coupling of the $5_{2}$ branes remains perturbative. ${ }^{\top 2}$ Acceptable values of $a$ tend to yield $\alpha_{s}\left(M_{Z}\right)$ slightly smaller than the preferred range. For $a=4$, Eq. (24) yields $\sin ^{2}\left(\theta_{W}\right)=0.23$ and $\alpha_{s}\left(M_{Z}\right)=0.11$.

\footnotetext{
${ }^{10} \mathrm{~A}$ related analysis with a similar conclusion was performed in [32].

${ }^{11}$ Note that in the embedding of the SM gauge group expected within the Shiu-Tye model (with the $S U(2)_{L, R}$ split between the two sets of branes), a similar analysis of the gauge couplings leads to a prediction of $g_{3}$ in the opposite direction.

${ }^{12}$ Choosing $a^{2}$ much larger than $O(10)$ leads to $\alpha_{5_{2}}=g_{5_{2}}^{2} / 4 \pi \gtrsim O(1)$ for reasonable ranges of the initial value of $\alpha_{5_{1}}=g_{5_{1}}^{2} / 4 \pi$.
} 


\section{B. Electroweak Symmetry Breaking}

We now turn to an investigation of radiative electroweak symmetry breaking within the 4224 model. As this model is a generalized Pati-Salam model, the embedding of the matter content on the branes does not distinguish between quarks and leptons (or between up and down type quarks) of a single generation, as they all reside within a given multiplet. The brane embedding is also such that third generation Yukawa unification is obtained at the string scale (with Yukawa couplings of $O(1)$ ), while the first and second generations get masses via higher-dimensional operators. Therefore, large $\tan \beta \equiv\left\langle H_{u}\right\rangle /\left\langle H_{d}\right\rangle \simeq m_{t} / m_{b} \sim$ $O(35-50)$ is required to produce the correct spectrum of SM fermions.

Radiative electroweak symmetry breaking in models with large $\tan \beta$ has been discussed extensively in the literature [33]. One can always choose within the bottom-up approach to fix $\mu$ and $b$ (or equivalently $B_{\mu} \equiv b / \mu$ ) by the electroweak symmetry breaking conditions. In the large $\tan \beta$ limit, the tree level minimization of the Higgs potential yields

$$
\begin{array}{r}
b \simeq \frac{1}{\tan \beta}\left[\left(m_{H_{d}}^{2}-m_{H_{u}}^{2}\right)-M_{Z}^{2}\right] \\
|\mu|^{2} \simeq-m_{H_{u}}^{2}-\frac{M_{Z}^{2}}{2} .
\end{array}
$$

The pseudoscalar mass-squared is given by

$$
m_{A}^{2}=\frac{b}{\sin 2 \beta} \simeq \frac{1}{2}\left[\left(m_{H_{d}}^{2}-m_{H_{u}}^{2}\right)-M_{Z}^{2}\right] .
$$

If the top and bottom Yukawas are comparable, then $m_{H_{u}}^{2}$ and $m_{H_{d}}^{2}$ will run similarly, so both $m_{H_{u}}^{2}$ and $m_{H_{d}}^{2}$ will run to negative values. Requiring the pseudoscalar mass-squared to be positive, we immediately obtain the result that $m_{H_{d}}^{2}=m_{H_{u}}^{2}=-M_{Z}^{2}$ is not allowed.

Although this seems problematic because the input values of $m_{H_{d}}^{2}$ and $m_{H_{u}}^{2}$ are equal within models in which the electroweak Higgs pair resides in a single GUT multiplet, these input values can be split by D term contributions (for a general discussion see [34) $m_{H_{d}}^{2}\left(M_{U}\right)-m_{H_{d}}^{2}\left(M_{U}\right)=4 g_{2}^{2} D$. These $\mathrm{D}$ term contributions to the soft masses of the electroweak Higgs doublets arise after the breaking of the Pati-Salam group and are proportional to the difference of the VEV's of the fields $H, \bar{H}$ which break the symmetry. As shown for Pati-Salam models [24], the D terms can be nonzero and of order the soft breaking scale. This analysis utilized a particular superpotential [22] which is symmetric under the exchange of $H$ and $\bar{H}$. This symmetry between $H$ and $\bar{H}$ is broken by their soft masses, leading to a $\mathrm{D}$ term which is proportional to their difference. In the 4224 model, since $H \rightarrow C_{1}^{5_{1}}$, $\bar{H} \rightarrow C_{2}^{5_{1}}$, and $\varphi_{1,2} \rightarrow C^{5_{1} 5_{2}}, D \simeq m_{3 / 2}^{2}\left(X_{0}^{2}-X_{3}^{2}\right)$. (The $\varphi_{1,2}$ fields do not contribute to the $\mathrm{D}$ term because their soft masses are identical). Thus, $m_{H_{d}}^{2}\left(M_{Z}\right)-m_{H_{u}}^{2}\left(M_{Z}\right) \sim m_{3 / 2}^{2} \gg M_{Z}^{2}$, consistent with radiative electroweak symmetry breaking.

While large $\tan \beta$ has certain desirable properties (such as large radiative corrections to the mass of the lightest neutral Higgs boson easily lift the mass to much larger values than the MSSM tree level result $m_{h}^{0} \simeq M_{Z}$ ), proper radiative electroweak symmetry breaking requires $\mu / \tan \beta \simeq B_{\mu}$, an apparent fine-tuning [35]. Within the top-down approach, this is a significant constraint in model-building. For example, this relation seems unnatural within string-motivated supergravity models, for which $\mu \sim B_{\mu} \sim m_{3 / 2}$ unless some cancellation or special alignment of Goldstino angles is present. 


\section{Low Energy Spectrum}

In this model, the low energy spectrum of the MSSM states is governed by an important sum rule related to the choice of a high string scale $\simeq 10^{16} \mathrm{GeV}$. In the gauge unification limit, $m_{3 / 2}^{2} \simeq\left(m_{3} / \sqrt{3} X_{1}\right)^{2}+O\left(1 / a^{2}\right)$ (using Eq. (7) ). From the expressions of soft masses and constraint $\sum_{i=0}^{3} X_{i}^{2}=1$, the following sum rule at the unification scale can be obtained:

$$
m_{h}^{2}+m_{\bar{F}_{3}}^{2}+m_{F_{3}}^{2}=m_{3}^{2}+O\left(1 / a^{2}\right) .
$$

This sum rule is familiar from the form of the soft supersymmetry breaking parameters within perturbative heterotic models. From the expression for the gaugino soft mass parameters, we see that by "decoupling" the effects of the $5_{2}$ brane sector to achieve gauge coupling unification, the gluino and bino masses approach the wino mass value $\sqrt{3} m_{3 / 2} X_{1} e^{-i \alpha_{1}}$ that they would have if only the $5_{1}$ sector was present. The soft parameters are similar to those from perturbative heterotic models, which resemble "single brane" Type I scenarios.

To study the implications of this sum rule, we must take into account the important requirement that the mass-squared eigenvalues of the sfermions must be positive to avoid charge or color breaking (CCB) minima. Positive input values for the soft superysmmetry breaking mass-squared parameters of the sfermions are also required, which implies that the Goldstino angles $X_{2}, X_{3}<1 / \sqrt{3}$ at the unification scale. The squark mass-squared eigenvalues always satisfy the positivity requirement because their diagonal terms run to larger values at low energies (driven strongly by $m_{3}$ ). In the slepton sector, given that $m_{\bar{F}_{3}}^{2}=m_{\tilde{\tau}_{R}}$ and $m_{F_{3}}^{2}=m_{\tilde{\tau}_{L}}$ do not substantially run from $M_{U}$ to $M_{Z}$ and the existence of large off-diagonal mixing for the third generation sfermions, $m_{\bar{F}_{3}}^{2}$ and $m_{F_{3}}^{2}$ must be quite large to satisfy the conditions for the absence of CCB minima. It is a generic prediction (from Eq. (29)) that $m_{3}\left(M_{U}\right)$ must be $(\geq 350) \mathrm{GeV}$. This yields a larger gluino mass $m_{3}\left(M_{Z}\right) \sim 1$ $\mathrm{TeV}$, disfavored from fine-tuning arguments [36].

In the large $\tan \beta$ limit, the tree level minimization condition of Higgs potential yields $|\mu|^{2} \simeq-m_{H_{u}}^{2}\left(M_{Z}\right)-M_{Z}^{2} / 2$. The renormalization group running of the soft mass parameters yields the relations $m_{H_{u}}^{2}\left(M_{Z}\right) \simeq m_{h}^{2}\left(M_{U}\right)-4 m_{3}^{2}\left(M_{U}\right)$ and $|\mu|^{2} \simeq 3 m_{3}^{2}\left(M_{U}\right)$. Therefore, proper radiative electroweak symmetry breaking requires $|\mu| \geq \sqrt{3} m_{3}\left(M_{U}\right)>600 \mathrm{GeV}$. This relatively large value of $\mu$ implies that the lightest two neutralino mass eignestates are bino and wino, with masses $m_{\chi_{1}^{0}} \simeq m_{3}\left(M_{U}\right) / 2 \geq 170 \mathrm{GeV}$ and $m_{\chi_{2}^{0}} \simeq m_{3}\left(M_{U}\right) \sim 350 \mathrm{GeV}$. The lightest chargino has the same mass as the neutral wino.

The squarks are generally rather heavy, with the lightest state around $600 \mathrm{GeV}$. $\tilde{\tau}_{1}$ is generally quite light due to the strong LR mixing. In a significant fraction of the parameter space, the lightest stau can be the lightest supersymmetric particle. It is desirable though that the lightest superpartner is neutral and constitutes the cold dark matter (CDM) of the universe, which yields an additional constraint $m_{\tilde{\tau}} \geq m_{\chi_{1}^{0}} \simeq m_{3}\left(M_{U}\right) / 2$. However, the

sum rule Eq. (29) implies $m_{\bar{F}_{3}}^{2}+m_{F_{3}}^{2} \leq m_{3}\left(M_{U}\right)^{2} \simeq 4 m_{\chi_{1}^{0}}^{2}$. Taking into account that the lightest mass of $\tilde{\tau}$ is a result of large off-diagonal mixing, it is difficult to make its mass larger than the mass of the lightest neutralino. This constraint forces the condition $m_{h} \sim 0$ (i.e. $\left.X_{0} \rightarrow 1 / \sqrt{3}\right)$. A particular choice of high energy parameters $X_{0}=0.58, X_{1}=0.79, X_{2}=0$, $m_{3 / 2}=318 \mathrm{GeV}, \alpha_{1,2}=0, a=\sqrt{10}$, and $\tan \beta=35$ yields the result $m_{\tilde{\tau}_{1}}=174 \mathrm{GeV}$ and $m_{\chi_{1}^{0}}=171 \mathrm{GeV}$. It is also important that the relic density produced by the LSP is within 
the range $\Omega h^{2} \leq 0.5$. Assuming the LSP mass is below the top threshold, we have checked that the relic density is below the experimental bound. The mass difference between the LSP and $\tilde{\tau}$ is very small so that coannihilation may become important. This will make the LSP relic density predicted in this model even smaller.

We now comment on the electric dipole moment (EDM) constraints. If phases are large and superpartner masses are small, the EDM's require cancellations [37 43] which are increasingly stringent for large $\tan \beta$. Although the superpartner masses are rather large in this model, they are not large enough to allow $O(1)$ phases without cancellations. Assuming that the soft trilinear couplings are given by Eq. (14) and that the phases take the values $\alpha_{0}=\alpha_{1}=\alpha_{2}=0$ ( $\alpha_{3}$ general), the gaugino masses are all real while only the $13,23,31$, and 32 elements of the soft trilinear couplings are complex. The EDMs are sensitive to the 11 entry of the LR blocks in the SCKM basis. With this choice of phases (and the phase of $\mu$ zero), the 4224 model is almost EDM-free because the 11 entries of the LR blocks are real. The rotation of the soft trilinear matrices to the SCKM basis does not reintroduce large CP violating phases to the 11 entry due to the factorizable structure of the $\tilde{A}$ terms [27, 44]. [3]

To summarize, the phenomenological pattern of the low energy mass spectrum is related via the sum rule to the following set of theoretical assumptions: (i) the high string and GUT breaking scales and (ii) only the MSSM states are present below the unification scale. As the Type I string scale is flexible, a natural question to ask is what results if the string and symmetry breaking scales are lowered e.g. to intermediate values (recall that semirealistic brane models have been constructed with nonsupersymmetric as well as supersymmetric sectors, for which the string scale naturally should be of order $10^{11} \mathrm{GeV}$ [14,15,94). Without a string-derived model, this depends on the assumed details of the symmetry breaking scale and the low energy particle content. In the 4224 model, the string scale cannot be lowered to intermediate scale values with only MSSM matter content. The model predicts $g_{3}^{2}=g_{2}^{2} /\left(1+1 / a^{2}\right)<g_{2}$ at the string scale, a condition violated by the RG running using MSSM beta functions and the low energy values of the gauge couplings. With additional matter there is flexibility to lower the scale while simultaneously lowering $a$ to deviate from the sum rule; this may be an interesting avenue for future exploration.

\section{CONCLUSIONS}

In this paper, we investigated aspects of the phenomenology of a Type I string-motivated model with two intersecting (orthogonal) stacks of five-branes with Pati-Salam gauge groups. The Pati-Salam $S U(4)_{c}$ is obtained from the diagonal subgroup of the $U(4)$ gauge groups from each set of branes, while $S U(2)_{L, R}$ are given by the $U(2)$ 's from one of the brane sectors. The third SM family is given by open string states from the $5_{1}$ sector which have an $O(1)$ Yukawa coupling to the electroweak Higgs doublets, while the first and second families are intersection states with zero Yukawa couplings at leading order. This 4224 model is similar

\footnotetext{
${ }^{13}$ However, it was recently pointed out that this feature does not necessarily hold in generic string models (in conjunction with obtaining a large CKM phase) [28].
} 
to the Shiu-Tye orientifold, but differs in the details of the SM gauge group embedding. The model has interesting features as well as generic problems which are summarized below.

An analysis of the diagonal breaking indicated that ensuring D flatness for the $U(N)$ gauge groups of the theory requires a doubling of the usual Higgs sectors needed for $S U(N)$ groups and predicts massless exotics at the renormalizable level. Within the 4224 model, this result leads to the presence of exotic colored states which render the proton unstable if they remain massless at higher orders. Decoupling the additional triplet pair in the PatiSalam symmetry breaking also is more subtle within $U(N)$ groups because the usual trilinear operators are forbidden by $U(1)$ gauge invariance. These issues signify generic challenges for string models which utilize intersecting D-branes in this manner.

Within the 4224 model, identifying the string scale to be near the usual GUT scale places significant constraints on the parameters of the theory. Obtaining proper low energy gauge couplings requires that one of the branes essentially decouples from the low energy physics (hence there is a hierarchy between the moduli VEVs which give the gauge couplings). In this effective single brane scenario, the soft terms are similar to those derived within perturbative heterotic models and obey a well-known sum rule. The constraints of the sum rule lead to a heavy superpartner spectrum and thus are not favored by fine-tuning arguments, but consistent radiative electroweak symmetry breaking can be achieved using the D term contributions to the soft scalar masses of the Higgs fields. Such constraints may be alleviated if the string scale is lowered, at the price of additional matter content.

\section{ACKNOWLEDGMENTS}

We thank M. Cvetič, G. Shiu, K. Dienes, and D. Chung for helpful discussions. We thank the Aspen Center for Physics for hospitality during several stages of this work. 


\section{REFERENCES}

[1] G. Pradisi and A. Sagnotti, Phys. Lett. B 216, 59 (1989); M. Bianchi and A. Sagnotti, Phys. Lett. B 247, 517 (1990); Nucl. Phys. B 361, 519 (1991); E. Gimon and J. Polchinski, Nucl. Phys. B 477 (1996) 715 hep-th/9601038; C. Angelantonj, M. Bianchi, G. Pradisi, A. Sagnotti and Ya.S. Stanev, Phys. Lett. B 385 (1996) 96 hep-th/9606169; Phys. Lett. B 387 (1996) 743.

[2] M. Berkooz and R. G. Leigh, Nucl. Phys. B 483 (1997) 187.

[3] G. Aldazabal, A. Font, L. Ibanez and G. Violero, Nucl. Phys. B 536, 29 (1998) hepth/9804026].

[4] Z. Kakushadze, G. Shiu and S.-H. Tye, Phys. Rev. D 58 (1998) 086001 hep-th/9803141; G. Zwart, Nucl. Phys. B 526 (1998) 378 [hep-th/9708040]; Z. Kakushadze, Nucl. Phys. B 512 (1998) 221 hep-th/9704059]; Z. Kakushadze and G. Shiu, Phys. Rev. D 56 (1997) 3686 [hep-th/9705163; Nucl. Phys. B 520 (1998) 75 hep-th/9706051]; L. Ibanez hepth/9802103]; D. O'Driscoll [hep-th/9801114]; J. Lykken, E. Poppitz, and S. Trivedi, Nucl. Phys. B 543 (1999) 105 [hep-th/9806080].

[5] G. Shiu and S.-H. Tye, Phys. Rev. D 58 (1998) 106007 hep-th/9805157.

[6] M. Cvetič, M. Plumacher, and J. Wang, hep-th/9911021]; M. Cvetic, A. Uranga and J. Wang,Nucl. Phys. B 595, 63 (2001).

[7] M. Klein and R. Rabadan, JHEP 0010, 049 (2000) hep-th/0008173.

[8] M. Cvetic, G. Shiu and A. Uranga, Phys. Rev. Lett. 87, 201801 (2001) hep-th/0107143; Nucl. Phys. B 615, 3 (2001) hep-th/0107166]; [hep-th/0111179.

[9] G. Aldazabal and A. Uranga, JHEP 9910, 024 (1999) hep-th/9908072]; G. Aldazabal, L. Ibanez and F. Quevedo, JHEP 0001, 031 (2000) hep-th/9909172].

[10] G. Aldazabal, S. Franco, L. Ibanez, R. Rabadan and A. Uranga, JHEP 0102, 047 (2001) hep-ph/0011132.

[11] J. C. Pati and A. Salam, Phys. Rev. D 10, 275 (1974).

[12] G. K. Leontaris and J. Rizos, Phys. Lett. B 510, 295 (2001) hep-ph/0012255.

[13] C. Burgess, L. Ibanez and F. Quevedo, Phys. Lett. B 447, 257 (1999) [hep-ph/9810535.

[14] G. Aldazabal, L. Ibanez, and F. Quevedo hep-ph/0001083; JHEP 0001:031, 2000, hepth/9909172; G. Aldazabal, L. Ibanez, F. Quevedo, and A. Uranga, hep-th/0005067.

[15] S. A. Abel, B. C. Allanach, F. Quevedo, L. Ibanez and M. Klein, JHEP0012, 026 (2000) hep-ph/0005260.

[16] L. Ibanez, C. Munoz, and S. Rigolin, Nucl. Phys. B 553 (1999) 43, hep-ph/9812397.

[17] K. Benakli, Phys. Lett. B 475, 77 (2000) [hep-ph/9911517.

[18] S. F. King and D. A. Rayner, hep-ph/0111333.

[19] L.Ibanez, R. Rabadan and A. Uranga, Nucl. Phys. B 542, 112 (1999) hep-th/9808139.

[20] M. A. Luty and W. I. Taylor, Phys. Rev. D 53, 3399 (1996) hep-th/9506098; T. Gherghetta, C. Kolda and S. P. Martin, Nucl. Phys. B 468, 37 (1996) hepph/9510370]; P. Binetruy, N. Irges, S. Lavignac and P. Ramond, Phys. Lett. B 403, 38 (1997) [hep-ph/9612442]; G. Cleaver, M. Cvetic, J. R. Espinosa, L. Everett and P. Langacker, Nucl. Phys. B 525, 3 (1998) hep-th/9711178.

[21] I. Antoniadis, G. K. Leontaris and J. Rizos, Phys. Lett. B 245, 161 (1990).

[22] S. F. King and Q. Shafi, Phys. Lett. B 422, 135 (1998) hep-ph/9711288.

[23] B. C. Allanach, S. F. King, G. K. Leontaris and S. Lola, Phys. Lett. B 407, 275 (1997) 
hep-ph/9703361; B. C. Allanach, S. F. King, G. K. Leontaris and S. Lola, Phys. Rev. D 56, 2632 (1997) hep-ph/9610517; B. C. Allanach and S. F. King, Nucl. Phys. B 459, 75 (1996) hep-ph/9509205; S. F. King, Phys. Lett. B 325, 129 (1994) [Erratum-ibid. B 325, 538 (1994)].

[24] S. F. King and M. Oliveira, Phys. Rev. D 63 (2001) 015010, hep-ph/0008183.

[25] S. F. King and M. Oliveira, Phys. Rev. D 63, 095004 (2001) hep-ph/0009287.

[26] A. Brignole, L. Ibanez and C. Munoz, Nucl. Phys. B 422, 125 (1994) [Erratum-ibid. B 436, 125 (1994)] [hep-ph/9308271]; A. Brignole, L. Ibanez, C. Munoz and C. Scheich, Z. Phys. C 74, 157 (1997) [hep-ph/9508258].

[27] S. Khalil, T. Kobayashi and O. Vives, Nucl. Phys. B 580, 275 (2000) hep-ph/0003086.

[28] S. Abel, S. Khalil and O. Lebedev, hep-ph/0112260; S. Khalil, O. Lebedev and S. Morris, hep-th/0110063; O. Lebedev, Phys. Lett. B 521, 71 (2001) hep-th/0108218.

[29] S. Khalil and T. Kobayashi, Phys. Lett. B 460, 341 (1999) hep-ph/9906374.

[30] G. F. Giudice and A. Masiero, Phys. Lett. B 206, 480 (1988).

[31] A. Donini and S. Rigolin, Nucl. Phys. B 550, 59 (1999) hep-ph/9901443.

[32] S. F. King and D. A. Rayner, Nucl. Phys. B 607, 77 (2001) [hep-ph/0012076].

[33] P. Langacker and N. Polonsky, Phys. Rev. D 49, 1454 (1994) hep-ph/9306205; ;S. Kelley, J. L. Lopez and D. V. Nanopoulos, Phys. Lett. B 274, 387 (1992); M. Carena, S. Pokorski and C. E. Wagner, Nucl. Phys. B 406, 59 (1993) hep-ph/9303202; B. C. Allanach and S. F. King, Phys. Lett. B 353, 477 (1995) hep-ph/9504406]; B. Ananthanarayan, G. Lazarides and Q. Shafi, Phys. Rev. D 44, 1613 (1991); G. L. Kane, C. Kolda, L. Roszkowski and J. D. Wells, Phys. Rev. D 49, 6173 (1994) hep-ph/9312272; M. Carena, M. Olechowski, S. Pokorski and C. E. Wagner, Nucl. Phys. B 426, 269 (1994) hep-ph/9402253]; L. J. Hall, R. Rattazzi and U. Sarid, Phys. Rev. D 50, 7048 (1994) hep-ph/9306309]; R. Rattazzi, U. Sarid and L. J. Hall, hep-ph/9405313; R. Rattazzi and U. Sarid, Phys. Rev. D 53, 1553 (1996) hep-ph/9505428; M. Olechowski and S. Pokorski, Phys. Lett. B 344, 201 (1995) [hep-ph/9407404; H. Baer, M. A. Diaz, J. Ferrandis and X. Tata, Phys. Rev. D 61, 111701 (2000) hep-ph/9907211.

[34] C. Kolda and S. P. Martin, Phys. Rev. D 53, 3871 (1996) hep-ph/9503445.

[35] A. E. Nelson and L. Randall, Phys. Lett. B 316, 516 (1993) hep-ph/9308277.

[36] G. L. Kane and S. F. King, Phys. Lett. B 451, 113 (1999).

[37] T. Falk and K. Olive, Phys. Lett. B 375, 196 (1996) hep-ph/9602299; Phys. Lett. B 439, 71 (1998) hep-ph/9806236.

[38] T. Ibrahim and P. Nath, Phys. Rev. D 57, 478 (1998) [Erratum-ibid. D 58, 019901 (1998)] hep-ph/9708456; Phys. Lett. B 418, 98 (1998) hep-ph/9707409]; Phys. Rev. D 58, 111301 (1998) [Erratum-ibid. D 60, 099902 (1998)] [hep-ph/9807501.

[39] M. Brhlik, G. Good and G. Kane, Phys. Rev. D 59, 115004 (1999) [hep-ph/9810457.

[40] D. Chang, W. Keung and A. Pilaftsis, Phys. Rev. Lett. 82, 900 (1999) [Erratum-ibid. 83, 3972 (1999)] hep-ph/9811202.

[41] T. Falk, K. Olive, M. Pospelov and R. Roiban, Nucl. Phys. B 560, 3 (1999) hepph/9904393.

[42] S. Pokorski, J. Rosiek and C. A. Savoy, Nucl. Phys. B 570, 81 (2000) hep-ph/9906206.

[43] S. Abel, S. Khalil and O. Lebedev, Phys. Rev. Lett. 86, 5850 (2001) [hep-ph/0103320;

Nucl. Phys. B 606, 151 (2001) hep-ph/0103031.

[44] T. Kobayashi and O. Vives, Phys. Lett. B 506, 323 (2001) hep-ph/0011200. 